\title{
Genomic and Molecular Mechanisms for Efficient Biodegradation of Aromatic
}

Dye

Su Sun ${ }^{\mathrm{a}, \mathrm{b}, \mathrm{c}, *}$, Shangxian Xie $\mathrm{e}^{\mathrm{a}, \mathrm{b}, \mathrm{d} *}$, Hu Chen ${ }^{\mathrm{a}, \mathrm{d}}$, Yanbing Cheng ${ }^{\mathrm{a}, \mathrm{d}}$, Yan Shi ${ }^{\mathrm{a}, \mathrm{d}}$, Xing Qin $^{\text {b }}$, Susie Y. Dai ${ }^{\text {a,c }}$, Xiaoyu Zhang ${ }^{\mathrm{b}, * *}$, Joshua S. Yuan ${ }^{\mathrm{a}, \mathrm{b}, \mathrm{d} * *}$

a Texas A\&M Agrilife Synthetic and Systems Biology Innovation Hub, and Institute for Plant Genomics and Biotechnology, Texas A\&M University, College Station, TX, USA

b School of Life Science and Technology, Huazhong University of Science and Technology, Wuhan, Hubei, China

${ }^{c}$ Department of Veterinary Pathology, Texas A\&M University, College station, TX, USA

${ }^{\mathrm{d}}$ Department of Plant Pathology and Microbiology, Texas A\&M University, College Station, TX, USA

*These authors contributed equally to this publication.

**For correspondence:

Joshua Yuan: syuan@tamu.edu

Xiaoyu Zhang: zhangxiaoyu@mail.hust.edu.cn

Mailing address: Joshua Yuan, 2123 TAMU, College Station, TX, 77843; Phone: $1-979-845-3016$. 


\section{Abstract}

Understanding the molecular mechanisms for aromatic compound degradation is crucial for the development of effective bioremediation strategies. We report the discovery of a novel phenomenon for improved degradation of Direct Red 5B azo dye by Irpex lacteus $\mathrm{CD} 2$ with lignin as a co-substrate. Transcriptomics analysis was performed to elucidate the molecular mechanisms of aromatic degradation in white rot fungus by comparing dye, lignin, and dye/lignin combined treatments. A full spectrum of lignin degradation peroxidases, oxidases, radical producing enzymes, and other relevant components were up-regulated under DR5B and lignin treatments. Lignin induced genes complemented the DR5B induced genes to provide essential enzymes and redox conditions for aromatic compound degradation. The transcriptomics analysis was further verified by manganese peroxidase $(\mathrm{MnP})$ protein over-expression, as revealed by proteomics, dye decolorization assay by purified MnP and increased hydroxyl radical levels, as indicated by an iron reducing activity assay. Overall, the molecular and genomic mechanisms indicated that effective aromatic polymer degradation requires synergistic enzymes and radical-mediated oxidative reactions to form an effective network of chemical processes. This study will help to guide the development of effective bioremediation and biomass degradation strategies.

Keyword: transcriptomics, molecular, mechanism, white rot fungi, azo dye. 


\section{Introduction}

Synthetic aromatic dyes are widely used for paper printing and textile dyes with more than $7 \times 10^{5}$ tons of annual global consumption. Azo dyes are the most widely used, yet they are also toxic to human, livestock and wildlife. Considering the strong toxicity, the bioremediation of azo dye with bacteria and fungi was extensively studied as an environmentally friendly, sustainable, and cost-effective approach for broad industrial applications [1]. Among the different microorganisms, white rot fungi belonging to basidiomycetes are distinct from other species for their outstanding capacity to degrade a large variety of recalcitrant pollutants [2, 3]. More importantly, some white rot fungal strains are also highly tolerant to toxic compounds, making them uniquely fit for the remediation of azo dyes [4]. Despite the potential, one of the major challenges of aromatic compound bioremediation is the limited understanding of the molecular mechanisms and environmental factors related to dye degradation.

Advanced "omics" technologies have been widely applied to study the mechanisms for various biological processes [3, 5-8]. Previous omics and chemical studies revealed a diverse group of enzymes and pathways involved in aromatic compound degradation. The degradation process normally involves both depolymerization and subsequent aromatic compound catabolism by microorganisms. Laccase, peroxidase, and many other enzymes are involved in the oxidation of the lignin macromolecule to depolymerize it into oligomers or monomers $[5,8,9]$. The non-enzymatic Fenton reaction complements enzymatic processes to further degrade the lignin 
macromolecule $[5,8,9]$. To understand the mechanisms of dye and lignin degradation, the patterns of these enzymes and their potential impacts on the chemical path of degradation need to be mapped out. In addition, the downstream aromatic compound catabolic pathways need to be characterized. Even with all the accumulated information, it is still unclear which pathways are important and how these pathways are regulated for dye degradation. Our discovery of enhanced azo dye Direct Red 5B (DR5B) (Figure 1A) decolorization by lignin provides a unique and effective system to dissect the mechanisms for both aromatic compound degradation and lignin catabolism.

In our study, we first quantified the enhanced decolorization of azo dye DR5B by the white rot fungus Irpex lacteus $\mathrm{CD} 2$ when alkali lignin was added as a co-substrate. Based on the analysis, comparative transcriptomics analysis was then carried out to elucidate the molecular and genomic mechanisms for both dye degradation and aromatic compound catabolism. The molecular mechanisms were further elucidated by proteomics analysis, an iron reducing activity assay, and the dye decolorization by MnP. The molecular and genomic mechanisms added new perspectives to our current understanding of aromatic compound degradation and could lead to new strategies to improve both dye remediation and lignin degradation.

\section{Materials and methods}

\subsection{Strain and chemicals}

The white rot fungus I. lacteus CD2 was previously studied and characterized for its 
highly efficient lignin degradation system [10]. The strain was originally isolated from rotted wood samples in Shennongjia Nature Reserve (Shennongjia, China) and maintained on potato dextrose agar (PDA) plates. The dye Direct Red 5B (CAS: 2610-11-9) has been commonly used in the textile industry and is widely used as a model compound for azo dyes in biodegradation research [11, 12]. The dye was purchased from Colorfran S.A. (Monterrey, Mexico). The alkali lignin (CAS: 8068-05-1) in this study was purchased from Sigma-Aldrich (St. Louis, Missouri, United States).

\subsection{DR5B decolorization and lignin co-metabolism}

Six $5 \mathrm{~mm}$ disks of $I$. Lacteus CD2 mycelium were cut from PDA plates and transferred to $100 \mathrm{ml}$ modified Kirk medium [13] in 250-ml Erlenmeyer flasks. The fungus was incubated at $28^{\circ} \mathrm{C}$ with a shaking speed of $120 \mathrm{rpm}$ before inoculation for decolorization treatments. The dye decolorization medium was prepared by adding $150 \mathrm{mg} \mathrm{L}^{-1}$ DR5B to Kirk medium supplemented with $1 \%$ glucose as an additional carbon source in the presence or absence of $0.03 \mathrm{~g} \mathrm{~L}^{-1}$ sterilized alkali lignin. Alkali lignin was sterilized using ultraviolet light for $30 \mathrm{~min}$. A $10.0 \%(\mathrm{v} / \mathrm{v})$ inoculum of fungus was then added and cultivated in cotton-plugged 250-ml flasks containing 100 $\mathrm{ml}$ of the aforementioned decolorization medium for six days. Experiments were performed in triplicate biological experiments. Mycelia-free supernatant was taken periodically for measurement of dye decolorization.

The maximum absorption wavelength of DR5B was determined by absorption 
spectrum scanning (190-800 nm) using an UV/VIS spectrophotometer. The decrease in absorbance was monitored using the spectrophotometer at the wavelength $510 \mathrm{~nm}$.

\subsection{Dye adsorption assay by fungal mycelia and lignin}

The DR5B adsorption rate by alkali lignin was measured by tracking the changes of $\mathrm{OD}_{510}$ with the UV/VIS Spectrophotometer in the same medium without fungus. The physical dye adsorption capacity of fungal mycelia during the DR5B decolorization process was detected by the following method: fungal mycelia from the dye decolorization medium were collected periodically by centrifugation at $7000 \mathrm{rpm}$ for $15 \mathrm{~min}$ and then soaked in $70 \%(\mathrm{v} / \mathrm{v})$ methanol for $24 \mathrm{~h}$ to recover the adsorbed dye. The recovered dye was measured by an UV/VIS Spectrophotometer at $510 \mathrm{~nm}$.

\subsection{Total RNA extraction and sequencing}

The fungal mycelium for RNA extraction was collected from I. lacteus CD grown in the following four different conditions after $72 \mathrm{~h}$ : Kirk medium only (CK), Kirk medium with $100 \mathrm{mg} \mathrm{L}^{-1}$ DR5B (CK+DR5B), Kirk medium with $0.03 \mathrm{~g} \mathrm{~L}^{-1}$ alkali lignin (CK+lignin), and Kirk medium with $100 \mathrm{mg} \mathrm{L}^{-1}$ DR5B and $0.03 \mathrm{~g} \mathrm{~L}^{-1}$ alkali lignin (CK+DR5B+lignin). Glucose was supplemented in all of the growth conditions to a final concentration of $1 \%$ as an additional carbon source. The fungal mycelia during the dye decolorization were harvested and immediately washed with RNase-free water, dried with a paper towel, and then ground in liquid nitrogen to a fine powder. RNA was extracted according to the manufacturer's manual for 
filamentous fungi in the Rneasy Plant Mini Kit (Qiagen Inc., TX, USA); the RNA concentration and quality were measured by bioanalyzer 2100 (Agilent Technologies, Santa Clara, CA) after RNA isolation. cDNA was synthesized and prepared for sequencing using the mRNA-Seq Sample Prep Kit (Illumina Inc., San Diego, USA). Sanger-read sequences of $100 \mathrm{bp}$ were generated using the Illumina Hiseq2500 platform.

\subsection{Transcriptome data de novo assembly}

High-quality clean reads were generated after removal of adapter sequences, duplication sequences, ambiguous reads (with more than 10\% Ns) and low-quality reads (with more than $50 \%$ bases whose quality was lower than 5). The Trinity program was used as recommended to obtain the best output of de novo assembly [14]; we integrated all reads from eight samples and applied Trinity with default parameters for single-end reads. CD-HIT (cut-off $=0.95$ ) was applied to generate more compact results from contigs longer than $200 \mathrm{bp}$ [15]. The Trinity analysis toolkit was again applied to predict ORFs (against Uniprot) for the Unigenes from the previous step [14]. Finally, we obtained 11845 Unigenes for the I. lacteus CD2 transcriptome.

\subsection{Functional annotation and classification of the assembled Unigenes}

Gene ontology (GO) annotations for the retained Unigenes were conducted using InterproScan (http://www.ebi.ac.uk/Tools/pfa/iprscan/). The output of InterproScan was then sent to the BGI WEGO online program (http://wego.genomics.org.cn) to 
generate an overview of the functional distribution of the transcriptome. FPKM (fragments per kilobase of transcript per million) using Trinity estimated the gene expression levels [14]. We used edgeR (p-value cut-off for FDR was 0.05, fold change was 2) to assess differential gene expression [16]. Functional enrichment analysis was performed by DAVID [17]. The results were considered significant if the false-detection rate (FDR) was less than $50 \%$ and the $P$ value was approximately 0.05 [17]. All genes (with detectable FPKM, i.e., greater than 0 in at least one sample) that could be assigned to the aforementioned functional categories were selected for downstream analysis. A heatmap was drawn using Cluster [18] and Treeview [19]. Signal peptide prediction of the proteins was conducted by SignalP4.0 [20] and Signal-3L [21].

\subsection{Shotgun proteomics analysis}

The fungal mycelia grown for $72 \mathrm{~h}$ in Kirk medium with $100 \mathrm{mg} \mathrm{L}^{-1}$ DR5B $(\mathrm{CK}+\mathrm{DR} 5 \mathrm{~B})$ and Kirk medium with $100 \mathrm{mg} \mathrm{L}^{-1} \mathrm{DR} 5 \mathrm{~B}$ and $0.03 \mathrm{~g} \mathrm{~L}^{-1}$ alkali lignin $(\mathrm{CK}+\mathrm{DR}$ 5B+Lignin) was collected and washed twice with double-distilled water. The collected mycelia was further dried with a paper towel and then ground into powder in liquid nitrogen. The sample was lysed by boiling for $10 \mathrm{~min}$ in alkaline-SDS buffer (5\% SDS, $0.15 \mathrm{M} \mathrm{NaCl}, 1 \mathrm{mM} \mathrm{MgCl} 2,0.1 \mathrm{mM}$ EDTA, $50 \mathrm{mM}$ pH 8.5 Tris-HCl, and 50 $\mathrm{mM}$ dithiothreitol). The supernatant was collected following centrifugation. The total protein was extracted by adding $100 \%$ trichloroacetic acid (TCA) to the supernatant to a final concentration of $20 \%$ and chilled at $-20^{\circ} \mathrm{C}$ for $2 \mathrm{~h}$. The protein was collected 
from the pellet and washed twice with chilled acetone. The collected protein was used for multidimensional protein identification technology (MudPIT)-based shotgun proteomics following the method described in our previous publication [22].

\subsection{DR5B dye decolorization assay by purified manganese peroxidase}

The MnP of I. lacteus $\mathrm{CD} 2$ was purified from the culture medium after the induced cultivation with $100 \mathrm{mg} / \mathrm{L}$ veratryl alcohol. The total extracellular protein of I. lacteus CD2 was pelletized by $80 \%$ ammonium sulfate at $4^{\circ} \mathrm{C}$. The collected protein was dissolved in sodium acetate buffer $(20 \mathrm{mM}, \mathrm{pH} 4.8)$. After the dialysis to remove ammonium sulfate, MnP was purified using DEAE Sepharose Fast Flow anion exchange column (GE) equilibrated with sodium acetate buffer (20 mM, pH 4.8). The DR5B decolorization assay using purified MnP was set up in a total volume of $1 \mathrm{~mL}$ buffer containing malonate $(20 \mathrm{mM}, \mathrm{pH} 4.5), \mathrm{Mn}^{2+}(1.6 \mathrm{mM}), \mathrm{H}_{2} \mathrm{O}_{2}(0.08 \mathrm{mM})$, along with the purified $\mathrm{MnP}(0.5 \mathrm{U} / \mathrm{ml})$ and DR5B dye $(100 \mathrm{mg} / \mathrm{L})$. The reaction mixture was incubated at $30^{\circ} \mathrm{C}$ for 1 hour and then measured by UV/VIS Spectrophotometer for dye decolorization effects.2.9. Measurement of iron-reducing activity

The iron-reducing activity in the DR5B dye decolorization system was measured based on the formation of a ferrozine- $\mathrm{Fe}^{2+}$ complex. Two $\mathrm{mL}$ of fugal supernatants from the aforementioned four conditions were collected by centrifugation. The supernatant was mixed with $0.5 \mathrm{~mL} \mathrm{FeCl} 3(1.2 \mathrm{mM})$ and $0.5 \mathrm{~mL}$ ferrozine $(15 \mathrm{mM})$. The absorbance change at $562 \mathrm{~nm}$ was detected immediately using a UV-visible spectrometer for $3 \mathrm{~min}$. One unit (A/min) of iron-reducing activity was defined as the 
rate of absorbance increase at $562 \mathrm{~nm}$ per minute.

\subsection{Data accessibility}

The Unigene assemblies for shot-gun transcriptomics were deposited at DDBJ/EMBL/GenBank under the accession number GBRE00000000. The version used in this paper is the first version with the accession number GBRE01000000. Individual sequences can be identified by a unique gene ID such as comp4780_c0 from the dataset. All of the raw sequencing data were also submitted to the same BioProject and can be accessed under SRA run accession number: SRR1577630, SRR1577727, $\quad$ SRR1577728, $\quad$ SRR1577729, $\quad$ SRR1577730, $\quad$ SRR1577731, SRR1577732, and SRR1577733.

The sequences of key genes discussed in this study can also be accessed with the GenBank accession numbers assigned for each sequence as follows: comp4779_c0 (KR296946); comp2101_c0 (KR296947); comp2416_c0 (KR296948); comp327_c0 (KR296949); comp4780_c0 (KR296950); comp2045_c0 (KR296951); comp2410_c0 (KR296952); comp8866_c0 (KR296953); comp4327_c0 (KR296954); comp5246_c0 (KR296955); comp5247_c0 (KR296956); comp5192_c0 (KR296957); comp3436_c0 (KR296958); comp586_c0 (KR296959); comp2569_c0 (KR296960); comp4778_c0 (KR296961); comp11117_c0 (KR296962); comp4781_c0 (KR296963); comp5188_c0 (KR296964).

\section{Result and discussion}

\subsection{Lignin as a co-substrate significantly promotes the decolorization of DR5B}


The use of alkali lignin (Figure 1B) as a co-substrate significantly improved the rate of DR5B decolorization. As shown in Figure 1C, the fungus I. lacteus CD2 was able to decolorize $82.62 \%$ of DR5B after $72 \mathrm{~h}$ in the presence of lignin compared with only $26.13 \%$ without lignin. To rule out decolorization caused by physical adsorption, we measured the dye adsorption rates of fungal mycelia or lignin using the method described in the Materials and Methods section. The adsorption assay showed that only $4.98 \%$ and $3.47 \%$ of dye was adsorbed after $72 \mathrm{~h}$ by fungal mycelia and lignin, respectively. These results suggested that the improved decolorization of DR5B upon lignin treatment was mainly due to the biochemical reactions rather than the physical adsorption (Figure 1D). In addition, the assay also showed that the adsorption of dye by fungal mycelia was significantly reduced after $48 \mathrm{~h}$ of incubation in presence of lignin (Figure 1D). These results clearly confirmed that the DR5B decolorization efficiency by I. lacteus CD2 could be significantly enhanced in the presence of lignin via the chemical reactions induced by lignin. This improved DR5B decolorization induced by lignin provided a novel and effective system to dissect the molecular mechanisms of lignin and dye degradation. On one hand, the lignin-induced dye decolorization can be exploited to design efficient fermentation conditions for dye-degradation. On the other hand, the results indicated that azo dye degradation depended on the molecular pathways evolved for lignin degradation, where the natural substrate stimulates more efficient degradation of azo dye. The comparative 'omics' analysis of different combinations of lignin and dye treatments will elucidate which enzymes and degradation pathways are specific for lignin degradation and 
which components are effective for dye degradation. Despite previous evidence of various co-substrates promoting aromatic compound degradation, few studies have focused on systems level studies of the mechanisms involved in the process. In particular, the lack of genome information for I. lacteus CD2 has hindered the investigation of systems and molecular mechanisms for efficient lignin and dye degradation. Comprehensive transcriptomics was carried out to address this challenge and elucidate the in-depth mechanisms for improved dye degradation by lignin.

\subsection{Overview of differential gene expression}

Comparative RNA-Seq transcriptomics analysis was carried out for I. lacteus CD2 growing on lignin, dye, the combination of lignin and dye, as well as control rich medium. Gene expression levels were estimated as FPKM using the Trinity program. The overview of gene expression patterns by cluster analysis revealed significant differential expression under various treatments. The four types of treatment led to 132 differentially expressed genes (Supplementary Table 1). As shown in Figure 2, the DR5B/lignin combined treatment specifically induced both extracellular poly-aromatic compound depolymerization enzymes such as manganese peroxidase (comp4779_c0) and aromatic compound catabolism genes such as homogentisate 1,2-dioxygenase (comp5246_c0 and comp5247_c0). The results indicated that those enzymes could account for more efficient DR5B decolorization in presence of lignin. The results also correlated well with gene ontology (GO) analysis in that the aromatic compound catabolic process was one of the most significantly enriched GOs during 
the dye and lignin combined treatment (Table 1).

\subsection{Enriched functional categories revealed the genomic mechanisms for improved dye decolorization by lignin}

GO analysis offers a semi-quantitative evaluation of biological processes and molecular functions involved in synergistic dye and lignin degradation. A total of 12 enriched functional categories were identified based on the SP-PIR keyword and GO term analysis of differentially expressed genes. Six different combinations of comparisons were analyzed for all of these enriched functional categories as shown in the 3D plot (Figure 3 and Supplementary Table 2). The results revealed at least two features relevant to dye and lignin degradation. The first feature was that several lignin and dye-degradation relevant categories were enriched with differentially expressed genes compared with other categories. These enriched categories included four types of GO terms relevant to dye and lignin metabolism. First, four GO categories were directly relevant to lignin and dye degradation, and these categories were lignin degradation, lignin catabolic process, aromatic compound catabolic process, and cellular aldehyde catabolic process. Second, three GO categories were relevant to the redox in the cell as well as the potential production and utilization of reactive oxygen species for lignin and dye degradation. These three categories were oxidation/reduction, oxidoreductase, and iron. Iron is known to be involved in the Fenton reaction to degrade lignin and aromatic polymers. The coordinate enrichment of iron relevant categories with redox-related categories was an indication of a 
potential Fenton reaction-type process for lignin and dye-degradation together with the enzymatic processes. Third, four GO categories were relevant to co-enzyme binding and production. Co-enzymes such as copper and heme are important for ligninases including manganese peroxidase $(\mathrm{MnP})$, lignin peroxidase, and laccase. Fourth, secretion processes and zymogens were enriched in the differentially regulated category. Zymogens include inactive enzyme precursors that can be activated when secreted. Secreted enzymes are important for lignin and dye degradation. Overall, most of the enriched categories were relevant to lignin and aromatic compound degradation, indicating a coordinative induction of redox enzymes and environment for dye and lignin degradation upon dye, lignin, and combined treatments.

The second feature was a clear synergy for dye and lignin treatments. As shown in Figure 1, lignin as a co-substrate significantly promoted dye decolorization. The analysis of functional categories provided genomic mechanisms for such synergy. As shown in Figure 3, the comparison of DR5B, lignin, and DR5B/ lignin combined treatment groups clearly showed that several relevant functional categories had the most number of differentially regulated genes in the co-substrate group. These categories included heme, heme-binding, oxidation reduction, oxidoreductase, iron, tetrapyrrole binding, aromatic compound catabolism, and aldehyde catabolism. The synergistic effects could be confirmed when comparing the DR5B/lignin combined treatment with the DR5B only treatment. Although DR5B treatment could lead to many differentially expressed genes in these categories, the combined treatment led to 14 
even more differentially regulated genes. The result indicated that the synergistic dye and lignin degradation might result from the stronger coordinative up-regulation of aromatic catabolism and oxidative enzymes. The comparison also identified many significantly differentially regulated genes in the aforementioned categories. Considering the enhanced effects on dye decoloration, the genes specifically differentially regulated with the DR5B/lignin combined treatment would be important to understand the molecular mechanisms for DR5B degradation.

\subsection{Molecular mechanisms for dye and alkali lignin depolymerization}

A detailed examination of differential gene and pathway expression suggested the unique mechanisms for dye degradation. Generally speaking, the lignin and aromatic dye degradation can be achieved by two separate steps. First, the heteropolymer of phenylpropane units needs to be depolymerized into monomeric or oligomeric aromatic compounds for uptake by fungal cells. Second, the aromatic monomers or oligomers need to be catabolized by the fungal cell. The first step often involves the oxygenated reactions assisted by extracellular enzymes. The functional category analysis indicated that oxidative enzymes, Fenton reaction components, and other relevant enzymes were differentially regulated in the DR5B, lignin, and co-substrate treatment. A detailed expression profile showed the overexpression of key enzymes involved in the aforementioned first step of dye and lignin degradation. As shown in Figure 4, a group of peroxidases and redox-regulating enzymes were significantly over-expressed in the DR5B/lignin combined treatment, which indicated their 
potentially critical roles in the depolymerization of aromatic compounds.

A detailed analysis revealed that those enzymes included three categories. The first category was the peroxidases including $\mathrm{MnP}$ and versatile peroxidases (VP). $\mathrm{MnP}$ is a group of classic peroxidases catalyzing the oxidation of a variety of lignin-related compounds including aromatic pollutants $[23,24]$. We found that several Unigenes encoding MnP (comp11117_c0, comp2569_c0, comp327_c0, comp4778_c0, comp4779_c0, comp4780_c0, and comp4781_c0) were highly expressed in lignin treatment or DR5B/lignin combined treatment (Figure 4), which indicated that MnP may be one of the major ligninolytic enzymes responsible for lignin degradation or dye decolorization in I. lacteus CD2. Besides MnP, versatile peroxidases were described as a new family of ligninolytic peroxidases, which had a hybrid molecular architecture that combined different oxidation sites with the heme cofactor for the oxidation of some high redox potential aromatic substrates [25]. Previous reports suggested that peroxidase synergized the redox condition and the Fenton reaction to maximize aromatic polymer degradation $[5,8,9]$. This study indicated a similar mechanism with specific refined enzymes.

The second category of enzymes induced by DR5B/lignin combined treatment was relevant to oxidative reactions and radical generation. These enzymes included alcohol oxidase (comp2410_c0), pyranose oxidase (comp586_c0), cellobiose dehydrogenase (comp4327_c0), choline dehydrogenase (comp2045_c0, comp2101_c0, comp2416_c0, comp3436_c0, comp5188_c0, comp5192_c0, and 
comp8866_c0), and others. Alcohol oxidase and pyranose oxidase reacted with abundant alcohol and glucose to generate reactive oxygen, which could in turn be used by peroxidase for lignin depolymerization [26]. Cellobiose dehydrogenase is involved in generating hydroxyl radicals for the Fenton reaction and also functions synergistically with manganese peroxidase in lignin degradation [27, 28]. Choline dehydrogenase was another group of enriched oxidoreductases under lignin treatment or DR5B/lignin combined treatment conditions. The enzyme might also play an important role in generating extracellular $\mathrm{H}_{2} \mathrm{O}_{2}$. In addition, most of these aforementioned enzymes have predicated secretion signal peptides (Figure 4). These enzymes clearly formed an oxidative network to generate and utilize reactive oxygen and other radicals for the purpose of dye decolorization and aromatic degradation. It was noticeable that the DR5B dye could induce only a few of these enzymes. Only lignin treatment or DR5B/lignin combined treatment could induce a more complete network of enzymes to achieve efficient dye degradation. These results were quite consistent with the previous study on the secretome of I. lacteus during wheat straw pretreatment, which also suggested that a redox biocatalytic system with $\mathrm{MnP}$ and $\mathrm{H}_{2} \mathrm{O}_{2}$ generation enzymes was critical for lignin oxidation [29].

The third category of up-regulated enzymes was several cytochrome P450s. Although the mechanisms remained elusive, cytochrome P450 enzymes were believed to be important in the degradation of low- and high-molecular-weight polycyclic aromatic hydrocarbons [30, 31]. 
In addition to these enzymes, the co-factors and other relevant processes were also enriched in the DR5B/lignin combined treatment samples. As shown in Figure 2 and Supplementary Table 2, the manganese transporter was specifically up-regulated in the combined treatment condition, synergizing the up-regulation of $\mathrm{MnP}$, indicating that $\mathrm{MnP}$ might play an important role in DR5B degradation. In addition to the transporter, many metal binding proteins were also up-regulated, providing an additional capacity for metal-mediated oxidation. The co-expression network analysis of the genes expressed under different conditions showed that most of the aforementioned genes were classified into same co-expressed module, which further supported our hypothesis (Supplementary Figure 2). Overall, the DR5B and lignin combined treatment induced a network of peroxidases, $\mathrm{H}_{2} \mathrm{O}_{2}$-generating enzymes, redox regulation enzymes, and other accessory enzymes to enable efficient oxidative reactions for dye and lignin degradation.

\subsection{The intracellular process for aromatic compound degradation}

The second step of aromatic polymer degradation is the catabolism of the aromatic compound monomer. Figure 5 showed that some enzymes in aromatic compound degradation pathways detected in I. lacteus CD2 based on the annotation of the transcriptomics data (Supplementary Table 3). Basically, most of the key branching pathways for lignin monomer degradation were found in the transcriptomics data, indicating a diverse and strong capacity for aromatic compound degradation by $I$. lacteus CD2. However, few of these genes were up-regulated in either lignin or 
DR5B/lignin combined treatment. The main gene up-regulated in the combined treatment was homogentisate 1,2-dioxygenase (comp5246_c0 and comp5247_c0), an important enzyme for opening aromatic rings for the next step of catabolism (Figure 5). In addition to aromatic compound degradation, many other genes in detoxification and relevant processes were up-regulated.

\subsection{Functional validation of transcriptomics-derived hypothesis by dye-decolorization assay of MnP, proteomics analysis and iron reducing activity assay}

The hypothesis derived from transcriptomics was validated from three aspects. First, to identify the abundance of MnP in dye decolorization, we carried out MudPIT-based shotgun proteomics to further verify the result of our transcriptomics analysis. The proteomics experiments will confirm the protein expression of the enzyme. The results showed that the protein level (as indicated by normalized peptide counts) of two major MnP enzymes (comp4779_c0 and comp4781_c0) found from transcriptomics were much more significantly induced in the DR5B/lignin combined treatment vs. the DR5B only treatment (Figure 6A and Supplementary Table 4). Second, the role of $\mathrm{MnP}$ in $\mathrm{DR} 5 \mathrm{~B}$ decolorization was validated by the dye degradation assay. The DR5B dye decolorization assay with purified MnP from $I$. lacteus CD2 suggested that the enzyme could efficiently degrade the azo dye (Figure 6B). Third, to verify if hydroxyl radicals were indeed induced to promote lignin or dye degradation, the iron reducing activity was carried out to serve as an important 
indicator for the hydroxyl radical level in fungal system. We measured the iron reducing activity of I. lacteus $\mathrm{CD} 2$ under different treatments (Figure 6C). As shown in Figure 6B, both DR5B and lignin induced a stronger iron reducing activity. More importantly, the DR5B/lignin combined treatment induced a much stronger iron reducing activity than all the treatments. The results indicated that the DR5B and lignin had a strong synergy that induced the production of free radicals to promote dye and lignin degradation. The result was highly consistent with the dye degradation experiments where lignin significantly promoted DR5B decolorization. The result was also well correlated with our systems biology analysis, where the DR5B/lignin combined treatment induced stronger expression of the peroxidases and radical-producing enzymes. Overall, the three aspects of data well validated the findings from transcriptomics analysis and suggested that a comprehensive enzymatic and chemical network was induced for the efficient degradation of DR5B in the presence of lignin.

\subsection{Conclusion}

Overall, the dye and lignin degradation in I. lacteus CD2 is carried out by a complex network of enzymes, irons, redox systems, and co-factors. The fungus I. lacteus CD2 could secrete several peroxidases ( $\mathrm{MnP}$ and $\mathrm{VP}$ ) and $\mathrm{H}_{2} \mathrm{O}_{2}$ generating oxidoreductases even without dye or alkali lignin induction (Figure 4, Supplementary Figure 1, and Supplementary Table 5). The baseline expression of peroxidases could depolymerize the dye and alkali lignin into small molecular compounds, which in turn could induce 
the more comprehensive degradation system. According to the transcriptomics analysis, the addition of dye DR5B could slightly enhance the expression of $\mathrm{H}_{2} \mathrm{O}_{2}$ generating oxidoreductases including alcohol oxidase, pyranose oxidase, and cellobiose dehydrogenase, as well as some aromatic compound degradation enzymes, such as homogentisate 1,2-dioxygenase. However, the DR5B treatment alone did not up-regulate, but rather slightly down-regulated, the expression of MnP and VP. This might be the primary reason why I. lacteus CD2 could not achieve high degradation efficiency for DR5B without lignin as the co-substrate. The lignin treatment significantly induced the expression of $\mathrm{MnP}$ and $\mathrm{VP}$, as well as that of metal binding and ion transporter related proteins, which could supply critical metal co-factors for MnP and VP. Dye decoloarization assay has verified the importance of MnP in degradation of DR5B. Meanwhile, lignin as a co-substrate enhanced the expression of extracellular oxidoreductases to generate more $\mathrm{H}_{2} \mathrm{O}_{2}$, which could maintain the redox cycling needed by MnP and VP. In addition to the lignolytic enzymes, the Fenton reaction based on hydroxyl radicals and iron might also play a critical role in DR5B and lignin depolymerization. The iron reducing assay helped to verify the results at certain degree. Lignolytic enzymes and chemical reactions networked together with downstream aromatic compound catabolism worked synergistically for the effective degradation of dye and lignin. Lignin, as a co-substrate for azo dye, induced the expression of some key components of this system to enable the rapid decolorization of azo dye.

\section{Acknowledgments}


The work in China was supported by a grant from the National Natural Science Foundation of China (31170104), the Major State Basic Research Development Program of China (2014CB138301) and the National High-tech Research and Development Program of China (2012AA101805). The work in the US was supported by US DOE EERE and Texas A\&M Agrilife Research's biofuel grant to JSY. 


\section{References}

[1] P. Singh, R. Sanghi, A. Pandey, L. Iyengar, Decolorization and partial degradation of monoazo dyes in sequential fixed-film anaerobic batch reactor (SFABR), Bioresour. Technol., 98 (2007) 2053-2056.

[2] L. Levin, L. Papinutti, F. Forchiassin, Evaluation of Argentinean white rot fungi for their ability to produce lignin-modifying enzymes and decolorize industrial dyes, Bioresour. Technol., 94 (2004) 169-176.

[3] S. Xie, R. Syrenne, S. Sun, J.S. Yuan, Exploration of Natural Biomass Utilization Systems (NBUS) for advanced biofuel-from systems biology to synthetic design, Curr. Opin. Biotechnol., 27 (2014) 195-203.

[4] J. Dittmann, W. Heyser, H. Bucking, Biodegradation of aromatic compounds by white rot and ectomycorrhizal fungal species and the accumulation of chlorinated benzoic acid in ectomycorrhizal pine seedlings, Chemosphere, 49 (2002) 297-306.

[5] D. Floudas, M. Binder, R. Riley, K. Barry, R.A. Blanchette, B. Henrissat, A.T. Martínez, R. Otillar, J.W. Spatafora, J.S. Yadav, A. Aerts, I. Benoit, A. Boyd, A. Carlson, A. Copeland, P.M. Coutinho, R.P. de Vries, P. Ferreira, K. Findley, B. Foster, J. Gaskell, D. Glotzer, P. Górecki, J. Heitman, C. Hesse, C. Hori, K. Igarashi, J.A. Jurgens, N. Kallen, P. Kersten, A. Kohler, U. Kües, T.K.A. Kumar, A. Kuo, K. LaButti, L.F. Larrondo, E. Lindquist, A. Ling, V. Lombard, S. Lucas, T. Lundell, R. Martin, D.J. McLaughlin, I. Morgenstern, E. Morin, C. Murat, L.G. Nagy, M. Nolan, R.A. Ohm, A. Patyshakuliyeva, A. Rokas, F.J. Ruiz-Dueñas, G. Sabat, A. Salamov, M. Samejima, J. Schmutz, J.C. Slot, F. St. John, J. Stenlid, H. Sun, S. Sun, K. Syed, A. Tsang, A. Wiebenga, D. Young, A. Pisabarro, D.C. Eastwood, F. Martin, D. Cullen, I.V. Grigoriev, D.S. Hibbett, The Paleozoic Origin of Enzymatic Lignin Decomposition Reconstructed from 31 Fungal Genomes, Science, 336 (2012) 1715-1719.

[6] J. Zajc, Y. Liu, W. Dai, Z. Yang, J. Hu, C. Gostincar, N. Gunde-Cimerman, Genome and transcriptome sequencing of the halophilic fungus Wallemia ichthyophaga: haloadaptations present and absent, BMC Genomics, 14 (2013) 617.

[7] X. Yu, S.P. Lund, R.A. Scott, J.W. Greenwald, A.H. Records, D. Nettleton, S.E. Lindow, D.C. Gross, G.A. Beattie, Transcriptional responses of Pseudomonas syringae to growth in epiphytic versus apoplastic leaf sites, Proc. Natl. Acad. Sci. U S A, 110 (2013) E425-434.

[8] S. Xie, X. Qin, Y. Cheng, D. Laskar, W. Qiao, S. Sun, L.H. Reyes, X. Wang, S.Y. Dai, S.E. Sattler, K. Kao, B. Yang, X. Zhang, J.S. Yuan, Simultaneous conversion of all cell wall components by an oleaginous fungus without chemi-physical pretreatment, Green Chem., 17 (2015) 1657-1667.

[9] E. Fernandez-Fueyo, F.J. Ruiz-Duenas, P. Ferreira, D. Floudas, D.S. Hibbett, P. Canessa, L.F. Larrondo, T.Y. James, D. Seelenfreund, S. Lobos, R. Polanco, M. Tello, Y. Honda, T. Watanabe, T. Watanabe, J.S. Ryu, C.P. Kubicek, M. Schmoll, J. Gaskell, K.E. Hammel, F.J. St John, A. Vanden Wymelenberg, G. Sabat, S. Splinter BonDurant, K. Syed, J.S. Yadav, H. Doddapaneni, V. Subramanian, J.L. Lavin, J.A. Oguiza, G. Perez, A.G. Pisabarro, L. Ramirez, F. Santoyo, E. Master, P.M. Coutinho, B. Henrissat, V. Lombard, J.K. Magnuson, U. Kues, C. Hori, K. Igarashi, M. Samejima, B.W. Held, K.W. Barry, K.M. LaButti, A. Lapidus, E.A. Lindquist, S.M. Lucas, R. Riley, A.A. Salamov, D. Hoffmeister, D. Schwenk, Y. Hadar, O. Yarden, R.P. de Vries, A. Wiebenga, J. Stenlid, D. Eastwood, I.V. Grigoriev, R.M. Berka, R.A. Blanchette, P. Kersten, A.T. Martinez, R. Vicuna, D. Cullen, Comparative genomics of Ceriporiopsis subvermispora and Phanerochaete chrysosporium provide insight into selective ligninolysis, Proc. Natl. Acad. Sci. U. S. A., 109 (2012) 5458-5463.

[10] H. Yu, W. Du, J. Zhang, F. Ma, X. Zhang, W. Zhong, Fungal treatment of cornstalks enhances the 
delignification and xylan loss during mild alkaline pretreatment and enzymatic digestibility of glucan, Bioresour. Technol., 101 (2010) 6728-6734.

[11] U.U. Jadhav, V.V. Dawkar, G.S. Ghodake, S.P. Govindwar, Biodegradation of Direct Red 5B, a textile dye by newly isolated Comamonas sp. UVS, J. Hazard. Mater., 158 (2008) 507-516.

[12] D.P. Tamboli, A.N. Kagalkar, M.U. Jadhav, J.P. Jadhav, S.P. Govindwar, Production of polyhydroxyhexadecanoic acid by using waste biomass of Sphingobacterium sp. ATM generated after degradation of textile dye Direct Red 5B, Bioresour. Technol., 101 (2010) 2421-2427.

[13] T.K. Kirk, E. Schultz, W.J. Connors, L.F. Lorenz, J.G. Zeikus, Influence of culture parameters on lignin metabolism byPhanerochaete chrysosporium, Arch. Microbiol., 117 (1978) 277-285.

[14] M.G. Grabherr, B.J. Haas, M. Yassour, J.Z. Levin, D.A. Thompson, I. Amit, X. Adiconis, L. Fan, R. Raychowdhury, Q. Zeng, Z. Chen, E. Mauceli, N. Hacohen, A. Gnirke, N. Rhind, F. di Palma, B.W. Birren, C. Nusbaum, K. Lindblad-Toh, N. Friedman, A. Regev, Full-length transcriptome assembly from RNA-Seq data without a reference genome, Nat. Biotechnol., 29 (2011) 644-652.

[15] L. Fu, B. Niu, Z. Zhu, S. Wu, W. Li, CD-HIT: accelerated for clustering the next-generation sequencing data, Bioinformatics, 28 (2012) 3150-3152.

[16] M.D. Robinson, D.J. McCarthy, G.K. Smyth, edgeR: a Bioconductor package for differential expression analysis of digital gene expression data, Bioinformatics, 26 (2010) 139-140.

[17] D.W. Huang, B.T. Sherman, R.A. Lempicki, Systematic and integrative analysis of large gene lists using DAVID bioinformatics resources, Nat. Protocols, 4 (2008) 44-57.

[18] M.B. Eisen, P.T. Spellman, P.O. Brown, D. Botstein, Cluster analysis and display of genome-wide expression patterns, Proc. Natl. Acad. Sci. U S A, 95 (1998) 14863-14868.

[19] R.D.M. Page, Tree View: An application to display phylogenetic trees on personal computers, Bioinformatics, 12 (1996) 357-358.

[20] T.N. Petersen, S. Brunak, G. von Heijne, H. Nielsen, SignalP 4.0: discriminating signal peptides from transmembrane regions, Nat. Methods, 8 (2011) 785-786.

[21] H.B. Shen, K.C. Chou, Signal-3L: A 3-layer approach for predicting signal peptides, Biochem. Biophys. Res. Commun., 363 (2007) 297-303.

[22] Y. Zhang, P. Gao, Z. Xing, S. Jin, Z. Chen, L. Liu, N. Constantino, X. Wang, W. Shi, J.S. Yuan, S.Y. Dai, Application of an Improved Proteomics Method for Abundant Protein Cleanup: Molecular and Genomic Mechanisms Study in Plant Defense, Mol. Cell. Proteomics, (2013).

[23] S. Sayadi, R. Ellouz, Roles of Lignin Peroxidase and Manganese Peroxidase from Phanerochaete chrysosporium in the Decolorization of Olive Mill Wastewaters, Appl. Environ. Microbiol., 61 (1995) 1098-1103.

[24] B.W. Bogan, B. Schoenike, R.T. Lamar, D. Cullen, Manganese peroxidase mRNA and enzyme activity levels during bioremediation of polycyclic aromatic hydrocarbon-contaminated soil with Phanerochaete chrysosporium, Appl. Environ. Microbiol., 62 (1996) 2381-2386.

[25] M. Perez-Boada, F.J. Ruiz-Duenas, R. Pogni, R. Basosi, T. Choinowski, M.J. Martinez, K. Piontek, A.T. Martinez, Versatile peroxidase oxidation of high redox potential aromatic compounds: site-directed mutagenesis, spectroscopic and crystallographic investigation of three long-range electron transfer pathways, J. Mol. Biol., 354 (2005) 385-402.

[26] P. Kersten, D. Cullen, Copper radical oxidases and related extracellular oxidoreductases of wood-decay Agaricomycetes, Fungal Genet. Biol., 72 (2014) 124-130.

[27] L. Hildén, G. Johansson, G. Pettersson, J. Li, P. Ljungquist, G. Henriksson, Do the extracellular enzymes cellobiose dehydrogenase and manganese peroxidase form a pathway in lignin 
biodegradation?, FEBS Letters, 477 (2000) 79-83.

[28] P. Kersten, D. Cullen, Extracellular oxidative systems of the lignin-degrading Basidiomycete Phanerochaete chrysosporium, Fungal Genet. Biol., 44 (2007) 77-87.

[29] D. Salvachua, A. Martinez, M. Tien, M. Lopez-Lucendo, F. Garcia, V. de los Rios, M. Martinez, A. Prieto, Differential proteomic analysis of the secretome of Irpex lacteus and other white-rot fungi during wheat straw pretreatment, Biotechnol. Biofuels, 6 (2013) 115.

[30] D. Martinez, L.F. Larrondo, N. Putnam, M.D. Gelpke, K. Huang, J. Chapman, K.G. Helfenbein, P. Ramaiya, J.C. Detter, F. Larimer, P.M. Coutinho, B. Henrissat, R. Berka, D. Cullen, D. Rokhsar, Genome sequence of the lignocellulose degrading fungus Phanerochaete chrysosporium strain RP78, Nature Biotechnology, 22 (2004) 695-700.

[31] H. Doddapaneni, V. Subramanian, B. Fu, D. Cullen, A comparative genomic analysis of the oxidative enzymes potentially involved in lignin degradation by Agaricus bisporus, Fungal Genet. Biol., 55 (2013) 22-31. 


\section{Table and figure captions}

Table 1. Overexpressed genes enriched in $\mathrm{CK}+\mathrm{DR} 5 \mathrm{~B}+$ lignin treatment samples.

Figure 1. Dye DR5B degradation by I. lacteus CD2. (a). Structure of dye DR5B. (b). Representative two-dimensional chemical structure of lignin. (c). Comparison of dye DR5B degradation efficiency by I. lacteus CD2 in the presence of alkali lignin (solid square) or in the absence of alkali lignin (solid circle).

Figure 2. Hierarchical cluster overview of differentially expressed genes among different treatments. All of the 132 differentially expressed Unigenes were presented in this heat map. Each row represented one Unigene and each column represented one treatment. $\mathrm{CK}, \mathrm{CK}+\mathrm{DR} 5 \mathrm{~B}, \mathrm{CK}+$ lignin and $\mathrm{CK}+\mathrm{DR}$ 5B+lignin referred to the same conditions described in the Materials and Methods section.

Figure 3. Comparison of functional category enrichment among the differentially expressed genes. The differentially expressed gene $(p$ value $<0.05$ and fold change $>$ 2) among the compared conditions was used for functional enrichment analysis. The $x$ axis indicates the functional categories which were significantly enriched in at least one pair of compared conditions (FDR $<50 \%$ and $p$ value $<0.05$ ). The $y$ axis indicates the compared treatment conditions as described in the Methods section. The $z$ axis indicates the count of the genes in each functional category. CK, CK+DR5B, $\mathrm{CK}+$ lignin and $\mathrm{CK}+\mathrm{DR}$ 5B+lignin referred to the same conditions described in the Materials and Methods section.

Figure 4. Cluster analysis of enzymes involved in lignin and aromatic compound degradation among different treatments. All of the Unigenes in this heatmap were 
annotated as belonging to Auxiliary Activities (AA) family 1 to AA family 8 enzymes using the CAZy database (E-value $\left.\leq 10^{6}\right)$. The Unigenes labeled with * indicated that their encoded proteins have predicted secretion signal peptides. Each row represented one Unigene, and each column represented one treatment. The expression value was normalized in each Unigene row. $\mathrm{CK}, \mathrm{CK}+\mathrm{DR} 5 \mathrm{~B}, \mathrm{CK}+$ lignin and $\mathrm{CK}+\mathrm{DR} 5 \mathrm{~B}+$ lignin referred to the same conditions described in the Materials and Methods section.

Figure 5. Catabolic pathways related to the degradation of the dye DR5B and alkali lignin derived compounds in I. lacteus CD2 according to the transcriptomics analysis. The black color indicates genes that were expected to be in 254 that were not yet identified in the transcriptomics data. The blue color indicates genes identified from transcriptomics analysis. The dark red color indicates genes that were differentially expressed and overexpressed in the dye and lignin treatments. The full gene names were listed in Supplementary table 3.

Figure 6. Relative expression level of two manganese peroxidase enzymes (A), decolorization of dye DR5B by purified $\mathrm{MnP}(\mathrm{B})$ and the iron-reducing activity (C) of I. lacteus $\mathrm{CD} 2$ under different treatment conditions. All of the error bars in this figure represent the standard deviation. $\mathrm{H}_{2} \mathrm{O}_{2}$ represented for the condition of incubating DR5B with $\mathrm{H}_{2} \mathrm{O}_{2}$ only as control; $\mathrm{H}_{2} \mathrm{O}_{2}+\mathrm{MnP}$ represented for the condition of incubating DR5B with $\mathrm{MnP}$ and $\mathrm{H}_{2} \mathrm{O}_{2} . \mathrm{CK}, \mathrm{CK}+\mathrm{DR} 5 \mathrm{~B}, \mathrm{CK}+$ lignin and $\mathrm{CK}+\mathrm{DR} 5 \mathrm{~B}+$ lignin referred to the same conditions described in the Materials and Methods section. 


\section{Tables and figures}

Table 1. Pathway enrichment of overexpressed genes in DR5B/lignin combined treatment samples.

\begin{tabular}{cccccc}
\hline Category & Term & $\%^{*}$ & P-Value & $\begin{array}{c}\text { Fold } \\
\text { Enrichment }\end{array}$ & FDR \\
\hline SP_PIR_KEYWORDS & Metal-binding & 54.55 & 0.008 & 3.72 & 0.08 \\
GOTERM_BP_FAT & $\begin{array}{c}\text { Aromatic compound } \\
\text { catabolic process }\end{array}$ & 18.18 & 0.061 & 28.26 & 0.50 \\
GOTERM_MF_FAT & $\begin{array}{c}\text { Transition metal ion } \\
\text { binding }\end{array}$ & 45.45 & 0.059 & 2.81 & 0.45 \\
\hline
\end{tabular}

*Percentage of the overexpressed genes in the enriched category 
A

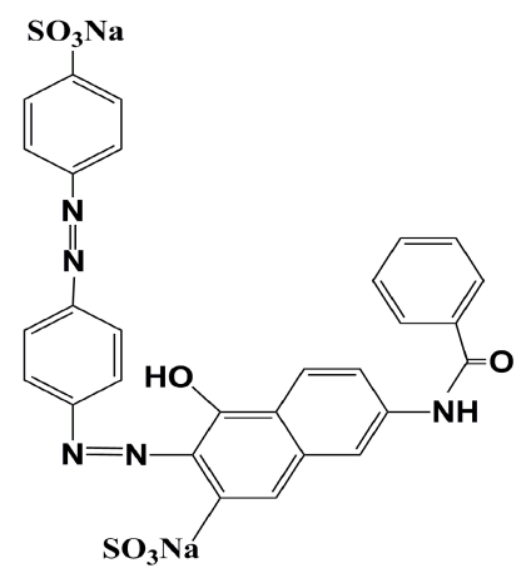

C

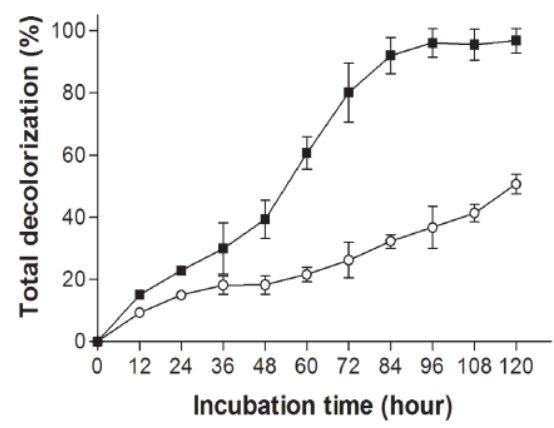

B

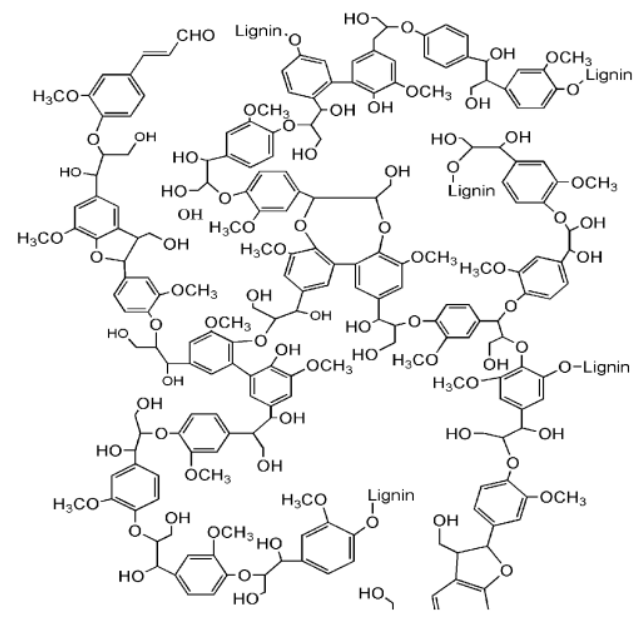

D

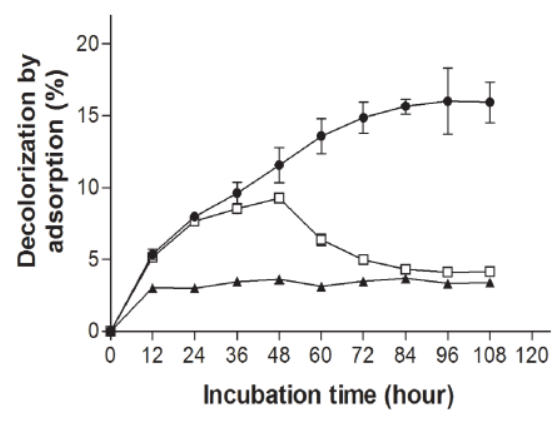

Figure 1. Dye DR5B degradation by I. lacteus CD2. (A). Structure of dye DR5B. (B). Representative two-dimensional chemical structure of lignin. (C). DR5B decolorization efficiency by I. lacteus CD2 in the presence of lignin (solid square) and without lignin (open circle). (D) DR5B adsorption by I. lacteus CD2 fungal mycelia in the presence of lignin (open square), fungal mycelia without lignin (solid circle), and lignin only (solid triangle). All of the error bars represent the standard deviation. 


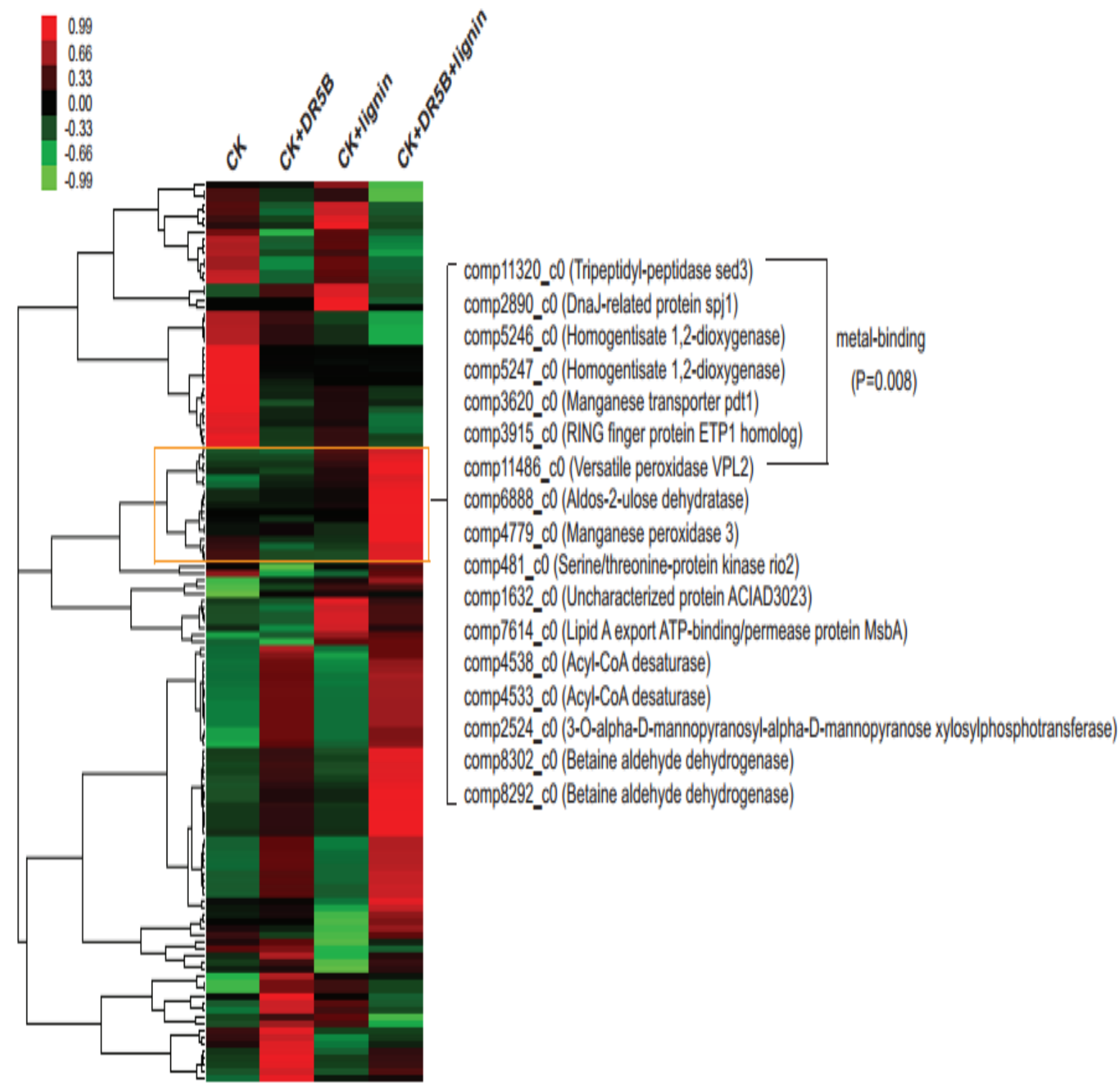

Figure 2. Hierarchical cluster overview of differentially expressed genes among different treatments. All of the 132 differentially expressed Unigenes were presented in this heat map. Each row represented one Unigene and each column represented one treatment. $\mathrm{CK}, \mathrm{CK}+\mathrm{DR} 5 \mathrm{~B}, \mathrm{CK}+$ lignin and $\mathrm{CK}+\mathrm{DR}$ 5B+lignin referred to the same conditions described in the Materials and Methods section. 


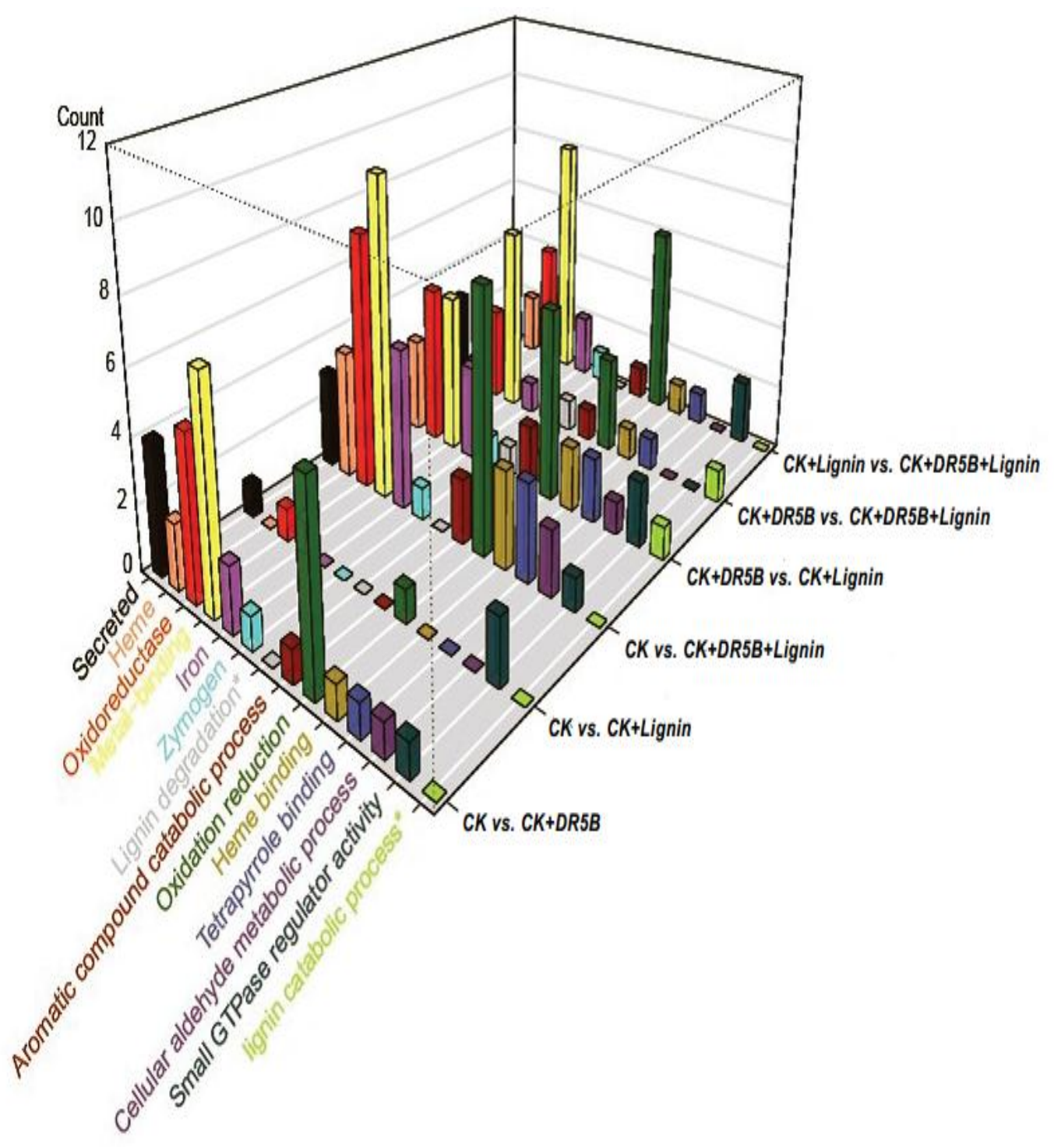

Figure 3. Comparison of functional category enrichment among the differentially expressed genes. The differentially expressed genes $(p$ value $<0.05$ and change fold $>$ 2) among the compared conditions were used for functional enrichment analysis. The $x$ axis indicates the functional categories which were significantly enriched in at least one pair of compared conditions (FDR $<50 \%$ and $p$ value $<0.05$ ). The $y$ axis indicates the compared treatment conditions as described in the Methods section. The $z$ axis indicates the count of the genes in each functional category. CK, CK+DR5B, $\mathrm{CK}+$ lignin and $\mathrm{CK}+\mathrm{DR} 5 \mathrm{~B}+$ lignin referred to the same conditions described in the Materials and Methods section. 


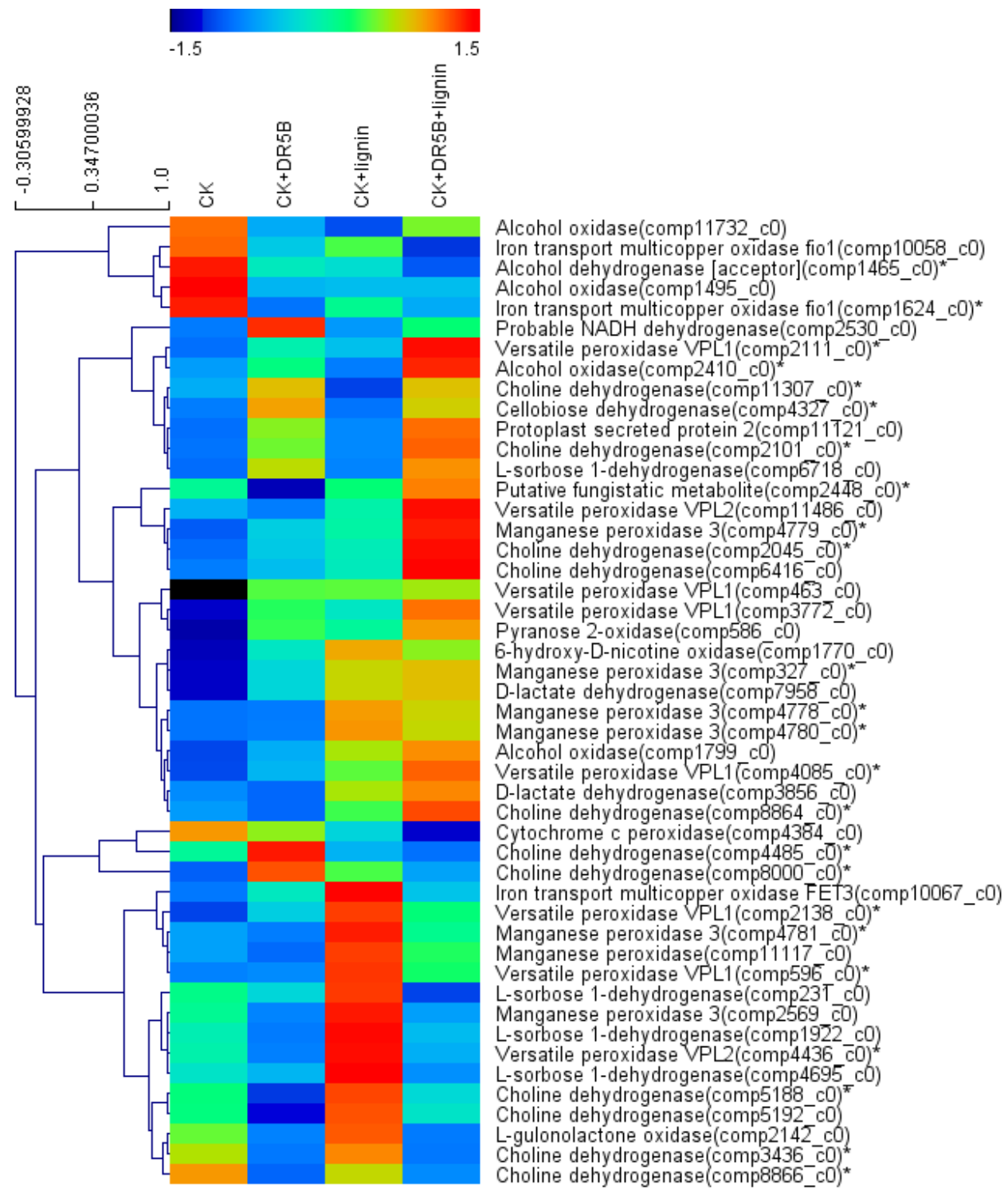

Figure 4. Cluster analysis of enzymes involved in lignin and aromatic compound degradation among different treatments. All of the Unigenes in this heatmap were annotated as belonging to Auxiliary Activities (AA) family 1 to AA family 8 enzymes using the CAZy database (E-value $\left.\leq 10^{6}\right)$. The Unigenes labeled with * indicated that their encoded proteins have predicted secretion signal peptides. Each row represented one Unigene, and each column represented one treatment. The expression value was normalized in each Unigene row. $\mathrm{CK}, \mathrm{CK}+\mathrm{DR} 5 \mathrm{~B}, \mathrm{CK}+$ lignin and $\mathrm{CK}+\mathrm{DR} 5 \mathrm{~B}+$ lignin referred to the same conditions described in the Materials and Methods section. 


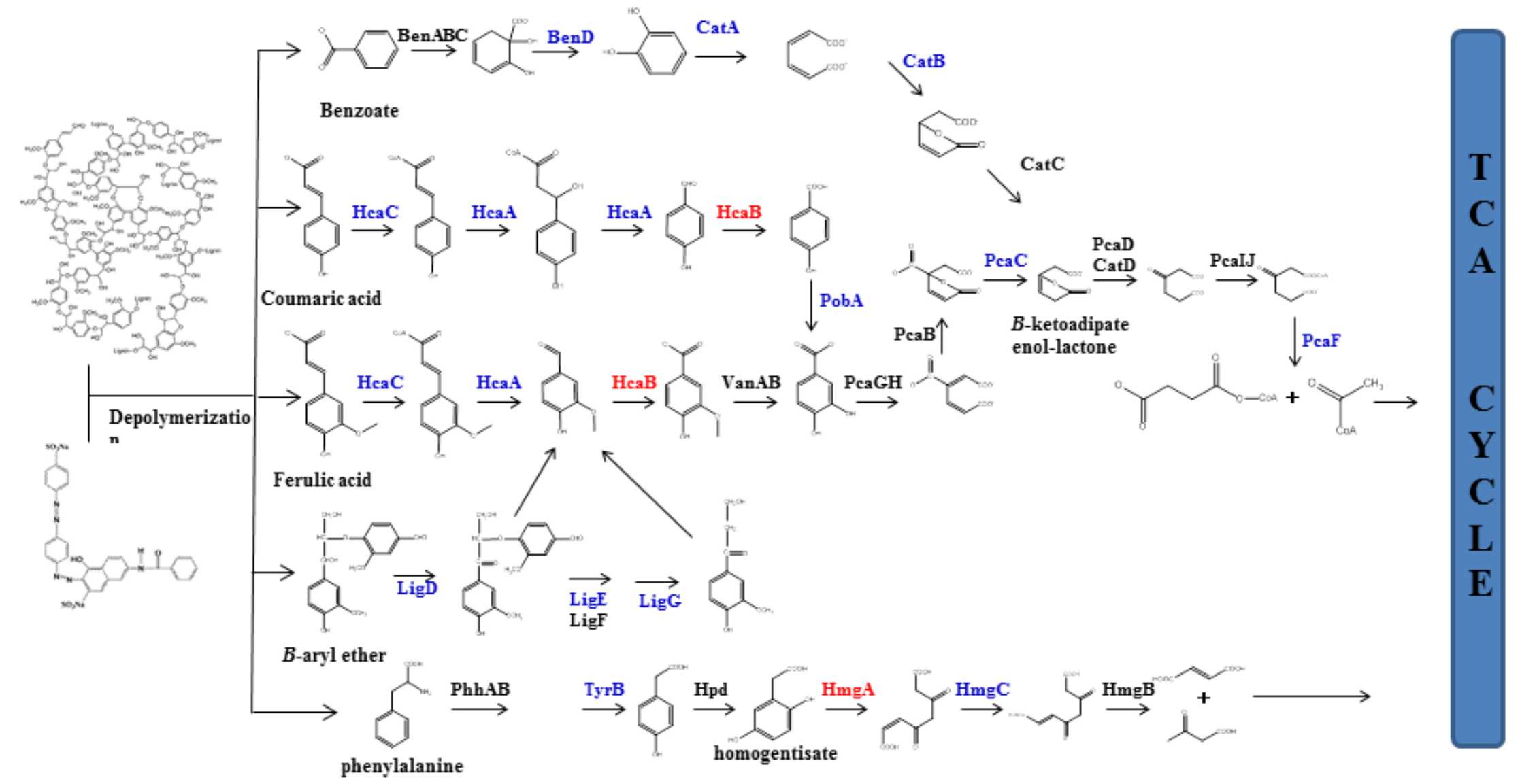


Figure 5. Catabolic pathways related to the degradation of dye DR5B and alkali lignin derived compounds in I. lacteus CD2 according to the transcriptomics analysis. The black color indicated genes that were expected to be in 254 , but were not yet identified in transcriptomics data. The blue color indicated genes identified from the transcriptomics analysis. The dark red color indicated genes differentially expressed and overexpressed in the dye and lignin treatments. The full gene names were listed in Supplementary table 3. 
A

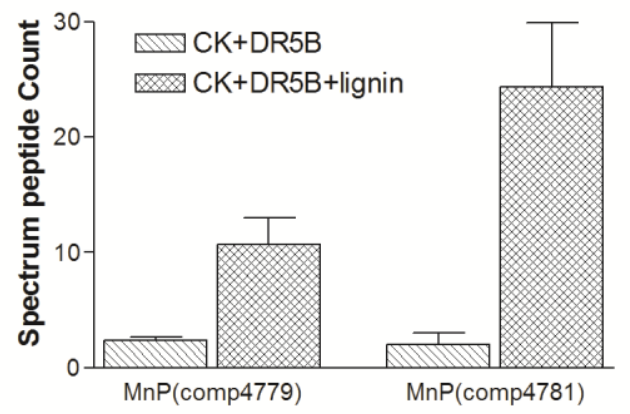

B

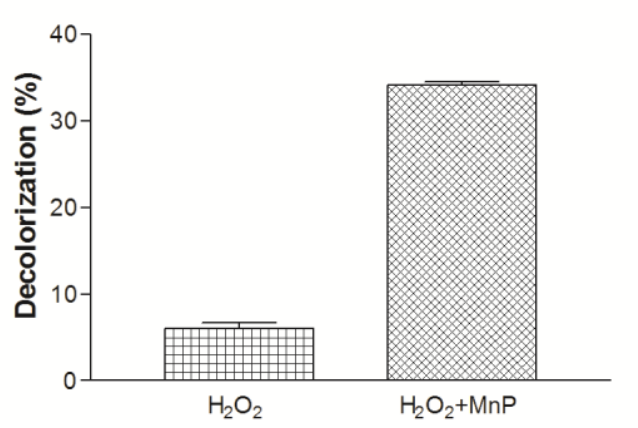

c

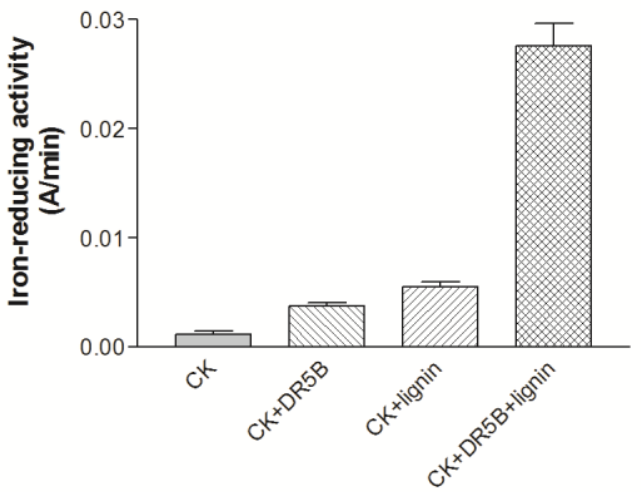

Figure 6. Relative expression level of two manganese peroxidase enzymes (A), decolorization of dye DR5B by purified MnP (B) and the iron-reducing activity (C) of I. lacteus CD2 under different treatment conditions. All of the error bars in this figure represent the standard deviation. $\mathrm{H}_{2} \mathrm{O}_{2}$ represented for the condition of incubating DR5B with $\mathrm{H}_{2} \mathrm{O}_{2}$ only as control; $\mathrm{H}_{2} \mathrm{O}_{2}+\mathrm{MnP}$ represented for the condition of incubating DR5B with $\mathrm{MnP}$ and $\mathrm{H}_{2} \mathrm{O}_{2}$. $\mathrm{CK}, \mathrm{CK}+\mathrm{DR} 5 \mathrm{~B}, \mathrm{CK}+$ lignin and $\mathrm{CK}+\mathrm{DR} 5 \mathrm{~B}+$ lignin referred to the same conditions described in the Materials and Methods section. 


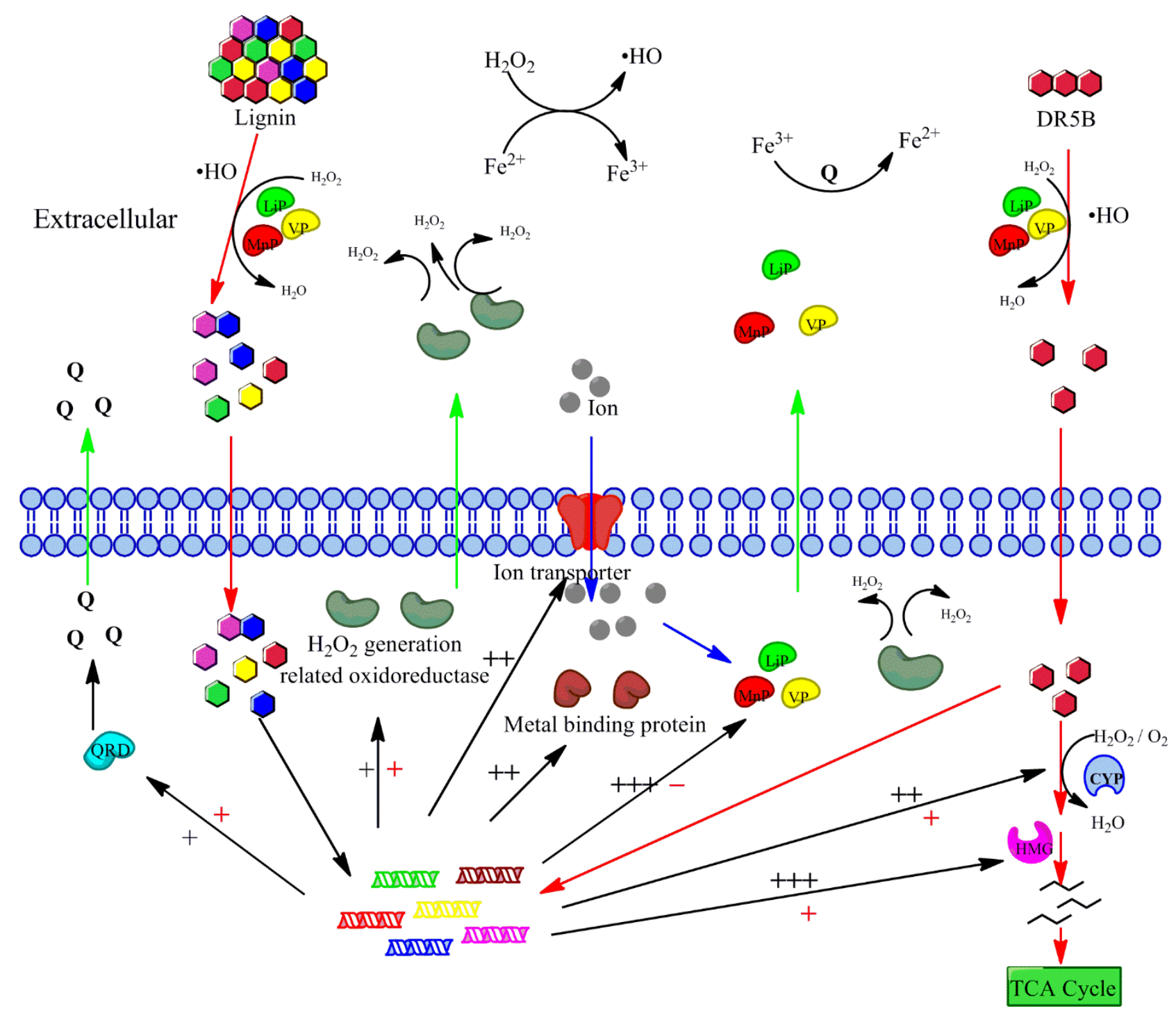

